\title{
FROM TLS POINT CLOUDS TO 3D MODELS OF TREES: A COMPARISON OF EXISTING ALGORITHMS FOR 3D TREE RECONSTRUCTION
}

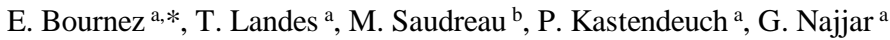 \\ ${ }^{a}$ ICube Laboratory UMR 7357, University of Strasbourg, CNRS, INSA Strasbourg, France - (elena.bournez, tania.landes)@insa- \\ strasbourg.fr; (kasten, georges.najjar)@unistra.fr \\ b PIAF Laboratory, UMR547 University Blaise Pascal, INRA Clermont-Ferrand, France - marc.saudreau@ clermont.inra.fr
}

\author{
Commission II
}

KEY WORDS: Trees, Terrestrial Laser Scanner, Point Cloud, 3D Model, Reconstruction, Algorithm, Assessment, Accuracy.

\begin{abstract}
:
3D models of tree geometry are important for numerous studies, such as for urban planning or agricultural studies. In climatology, tree models can be necessary for simulating the cooling effect of trees by estimating their evapotranspiration. The literature shows that the more accurate the 3D structure of a tree is, the more accurate microclimate models are. This is the reason why, since 2013, we have been developing an algorithm for the reconstruction of trees from terrestrial laser scanner (TLS) data, which we call TreeArchitecture. Meanwhile, new promising algorithms dedicated to tree reconstruction have emerged in the literature. In this paper, we assess the capacity of our algorithm and of two others -PlantScan3D and SimpleTree- to reconstruct the 3D structure of trees. The aim of this reconstruction is to be able to characterize the geometric complexity of trees, with different heights, sizes and shapes of branches. Based on a specific surveying workflow with a TLS, we have acquired dense point clouds of six different urban trees, with specific architectures, before reconstructing them with each algorithm. Finally, qualitative and quantitative assessments of the models are performed using reference tree reconstructions and field measurements. Based on this assessment, the advantages and the limits of every reconstruction algorithm are highlighted. Anyway, very satisfying results can be reached for 3D reconstructions of tree topology as well as of tree volume.
\end{abstract}

\section{INTRODUCTION}

3D models of trees, with leaves and wood, are useful for a wide range of applications. Landscapes created in video games need tree representations in order to be more realistic. In urban planning projects the integration of tree models is required for improving the pedestrian point of view. In agriculture projects, detailed models of trees are also required for monitoring or planning the tree growth. In our project, we need models of trees in the context of urban climatology. The cooling effect of individual trees can be simulated with ecophysiological models, such as the model RATP (Sinoquet et al., 2001). Based on the exact geometry of a tree, this kind of microclimate model is able to simulate the level of evapotranspiration produced by the tree (Bournez et al., 2016).

The 3D reconstruction of trees is a very challenging task given the complex geometry of trees. Trees are generally composed of leaves and several orders of branches with different sizes and diameters. The reconstruction of tree wood components is even more complex if the tree is under leaf-on conditions and if it is not regularly pruned. Leaves hide branches and if the tree is not pruned much thinner branches appear in several different directions. Moreover, even in urban environments, an important diversity of tree species and therefore of tree geometries might occur. These observations highlight the difficulty to acquire detailed 3D information on the geometry and to create reliable 3D models of trees. As branches and shoots bear leaves, an accurate modeling purpose may rely on the reconstruction of the tree wood structure, before spatializing the leaves in the crown.
This is the reason why, in this paper, trees without leaves are considered.

Aim of this study is to find the most appropriate method for accurately reconstructing, as automatically as possible, an individual tree from point clouds in order to use it in an ecophysiological model. For this purpose, we compare the 3D reconstruction algorithm, developed in our laboratory, with two other open source algorithms. The assessment is carried out on the use of the algorithms and on their results. These algorithms were tested on different geometries of trees.

First, a state of the art describes techniques usually used for acquiring tree point clouds as well as methods developed for 3D tree reconstruction. Then, trees under study and the tested reconstruction algorithms are presented. Afterwards, results for each tree obtained with each algorithm and qualitative and quantitative assessments of these results are given. Finally, the evaluation on the possibility, with these algorithms, to reconstruct trees in $3 \mathrm{D}$ is exposed.

\section{RELATED WORKS}

\subsection{Acquisition methods of tree geometry}

In the literature, three main techniques are used for acquiring 3D point clouds of trees: manual electromagnetic digitizing, photogrammetric and laser scanning techniques. The first one makes it possible to acquire several points directly on the wood of the tree, but requires contact with the object (Sinoquet and Rivet, 1997). Although this technique is adapted for accurate

\footnotetext{
* Corresponding author
} 
reconstructions, the acquisition step remains manual and tedious. The two last methods are contactless automatic acquisition techniques. Based on terrestrial photographs, a point cloud of the tree can be generated by dense image matching (Quan et al., 2006). A drawback of the photogrammetric technique is that a huge number of points of view is required in order to obtain enough data for the reconstruction of only one tree. Finally, the terrestrial laser scanning (TLS) technique seems to be the most adequate solution (van Leeuwen and Nieuwenhuis, 2010). Indeed, TLS allows a fast and accurate acquisition of dense point clouds, which is necessary for the purpose of obtaining a complete and detailed model of trees.

The quality of the point cloud covering an object depends on errors related to the scanned object, instrumental errors, environmental errors and methodological (Grussenmeyer et al., 2016). Errors related to the scanned tree are explained by its geometric complexity: topology, number and thickness of branches. Noise in the point cloud is the consequence of thin branches, whereas holes are produced by masks created by large and dense branching structures. The point cloud quality also depends on the conditions of field acquisition such as for instance the distance between trees and stations, the number of stations, the setting of the TLS and the weather conditions. In most studies, the acquisition method carried out in the field with a laser scanner consists in establishing several stations around the trees. The higher the number of stations around a tree, the lower the number of holes in the tree point cloud. In order to have enough points for characterizing branches, the point spacing must be defined depending on the diameter of the branches. Moreover, artefacts can occur by windy conditions. Finally, the processing steps of the raw point clouds, i.e. registration, segmentation, sampling or de-noising might also affect the point cloud quality. This error budget must be considered in the assessment of the 3D point cloud of a tree which will be used for tree $3 \mathrm{D}$ reconstruction.

\subsection{Accurate 3D reconstruction methods of trees}

Several methods for the 3D reconstruction of trees from point clouds emerge from the literature. If only a realistic model is necessary, the meshing solution is suggested. However, we put aside this method since the generated data is heavy and the interpretation of tree characteristics, such as length, orientation or hierarchy of branches, is rather difficult except for the volume of the biomass.

Two other categories of methods produce reconstructions which enable the extraction of previously mentioned tree characteristics.

The first category of methods uses the skeleton concept for describing the geometry of trees. A skeleton is a wireframe model of the tree, characterized by zero thickness. It is included and centered in the shape formed by the point cloud. The main advantage of a skeleton is that it enables connectivity and it preserves topology between branches (Cornea et al., 2005). Several processes of skeletonization for tree reconstruction have been developed in the literature. Some methods carry out firstly a contraction, before extracting a skeleton from the reduced point cloud (Cao et al., 2010; Preuksakarn, 2012). Others transform the point cloud into a graph in order to organize it for obtaining the skeleton (Côté et al., 2009; Livny et al., 2010; Xu et al., 2007). We can also find a method which segments the point cloud in octree before computing the centroid of each cell to form the skeleton (Bucksch et al., 2010). Moreover, based on a branch skeleton, it is possible to reconstruct its thickness and then its volume, by a previous determination of the branch diameter (Côté et al., 2009; Livny et al., 2010; Preuksakarn, 2012; Xu et al., 2007).

The second category of methods for tree reconstruction is based on the adjustment of the tree point cloud by geometric primitives such as cylinders, cones, spheres, etc. The cylinder seems to be the most appropriate primitive (Rahman et al., 2015) since it allows the production of a realistic model and the extraction of geometric characteristics. With this kind of model, the connectivity and the topology of branches are also preserved and the volume of the wood can be determined. Some existing methods perform a segmentation of the point cloud in sections in order to use these sections for cylinder adjustments (Pfeifer et al., 2004; Raumonen et al., 2013). Another method uses spheres in order to follow the geometry of the tree and to extract the skeleton and the thickness of branches for creating cylinders (Hackenberg et al., 2014).

Thus, the skeletonization and the adjustment of primitive methods allow respectively an indirect or a direct reconstruction of trees as a volumetric 3D model. These methods are integrated in the algorithms we would like to test. Among the work reported in the literature, two algorithms emerge particularly because of the results they might provide for $3 \mathrm{D}$ tree reconstruction and because they are open source. Their results will be compared to our own 3D reconstruction algorithm.

\section{DATA AND ALGORITHMS USED}

Aim of our study is to evaluate the accuracy of several tree reconstruction algorithms with several tree point clouds acquired with a terrestrial laser scanner.

\subsection{Algorithms used}

The first algorithm, called "TreeArchitecture" (Landes et al., 2015), has been developed in our laboratory (ICube) for climatological studies in order to simulate the tree impact on the urban climate in terms of evapotranspiration. TreeArchitecture uses the method of Cao et al. (2010) to create a raw skeleton of trees. Based on the point cloud, a geometric contraction followed by a skeletonization using the Delaunay triangulation are carried out. After that, the raw skeleton, composed of several segments, is extended at its extremities to guarantee the proper size of the branches. Moreover, each point is classified and ordered according to the component (branch or trunk) it belongs to. Afterwards, the volumetric model of each segment is reconstructed by mean branch diameter determination. TreeArchitecture is written in Matlab.

The second algorithm, known as "PlantScan3D" (Boudon et al., 2014), has been developed in the INRIA laboratory. It was created in a context of biology and agronomy for generating quantitative models of plant development and for comparing different methods of tree reconstruction implemented inside. For our test we have chosen the skeletonization method of $\mathrm{Xu}$ et al. (2007) which seems to provide realistic results and which differs from our method. This method consists in connecting the points of the point cloud to form a graph. Then, by a computation of distance, the algorithm produces a skeleton ordered as a "Branch Structure Graph" (BSG). This BSG format allows us to keep the topology of the branch structure. Afterwards, among the different methods proposed in the tool for reconstructing the volume of branches, we have chosen that which computes the mean 
diameter of each part of the skeleton. This method seems to provide better results than the others that were implemented. The open source version of PlantScan3D used in this paper is the 6.0 (June the $17^{\text {th }}, 2016$ ) and it is written in $\mathrm{C}++$ and Python.

The third algorithm tested in this work is "SimpleTree", developed by Hackenberg et al. (2015). It was created to build quantitative structure models (QSM) of trees for forestry applications. This type of model allows the quantification of the above ground biomass in a hierarchical order. The volumetric model, created by SimpleTree, involves a fitting of cylinders by using the idea of cutting sphere surfaces. These spheres allow the extraction of the skeleton nodes and the thickness of the point cloud cut by each sphere. Thus, it will be interesting to compare this method of reconstruction to the others which are totally different. SimpleTree is written in $\mathrm{C}++$ and we used the version available in http://www.simpletree.uni-freiburg.de/ supported by Linux Ubuntu.

Based on a sample of tree point clouds, we will assess the possibility to reconstruct trees accurately thanks to these algorithms.

\subsection{Trees under study}

The trees selected to assess the reconstruction algorithms are located in the city of Strasbourg, France.

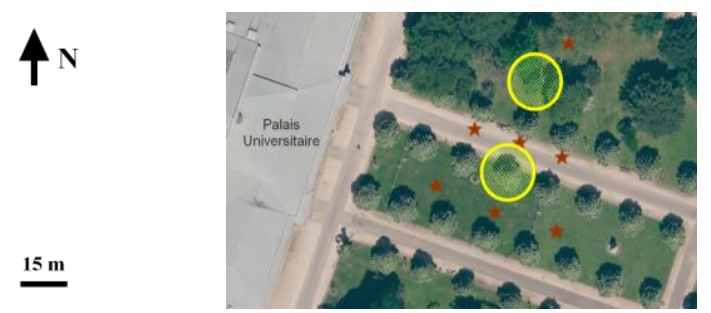

(a)

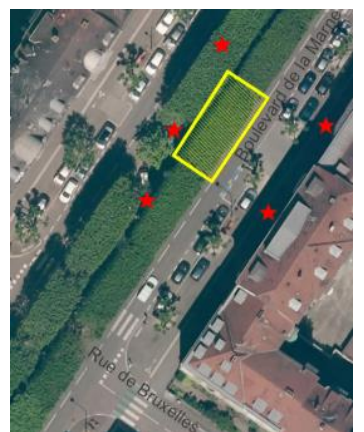

(b)

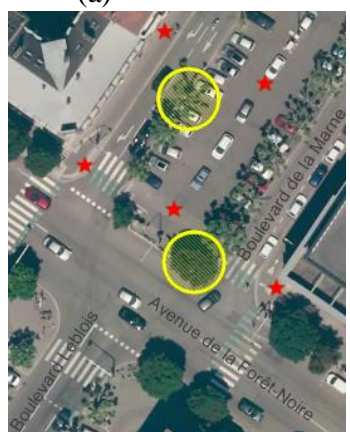

(c)
Figure 1: Trees captured by terrestrial laser scanning (in yellow) and the TLS station locations (red stars) in different areas in the

city of Strasbourg; a) garden of Palais-U; b) and c) Marne

Boulevard (Images: Courtesy of the Strasbourg Eurometropolis).

A field measurement campaign with a TLS (FARO Focus 3D X 330) was carried out in three different urban areas (Figure 1), where several species and geometries of trees can be found. The first area, located in a public garden, contains two rows of aligned trees which are regularly spaced (Figure 1a), whereas in the second and the third area we can find street trees, aligned but not isolated, surrounded by buildings (Figure $1 \mathrm{~b}$ and Figure 1c). For each area, the acquisition process was the same, with three or five TLS stations around trees (red stars in Figure 1). The instrument settings were a field of view of $300^{\circ} / 360^{\circ}$, the capture of color images and a space between points depending on the distance to the tree and on the thickness of the branches $(6 \mathrm{~mm}$ or
$12 \mathrm{~mm}$ at $10 \mathrm{~m})$. After that, the acquired data were preprocessed, i.e. the steps of registration, georeferencing, segmentation and de-noising were applied to obtain an adequate point cloud for the future 3D reconstruction. A sampling of a minimal distance of $1 \mathrm{~cm}$ and a reduction in the number of points of more than $50 \%$ were applied to each point cloud. This preprocessing is necessary for our algorithm which cannot operate with large amount of data and also for reducing the processing time of the tests. Final point clouds contained less noise, but to the detriment of small details of tree geometries.

Six different trees, illustrated in Figure 3, were selected: one pruned silver lime tree, one pruned silver lime tree with shoots, one pruned chestnut tree, two not pruned chestnut trees and a pruned plane tree. They were chosen for their geometric specificities, as described in Table 2: tree size, topology, number and thickness of branches, etc. This sample of trees is used for assessing the performance of the reconstruction algorithms for reconstructing pruned and unpruned trees, small and tall trees, thin and large branches, intertwined and not intertwined branches and also spaced and close branches. More exactly, the pruned silver lime tree is the standard case, i.e. it is characterized by spaced, not intertwined and large branches (Figure 3b). The chestnut tree 2 was also chosen for its simple geometry, but it is not pruned and it has thin branches (Figure 3c). The chestnut tree 1 (Figure 3a) is, from a geometric point of view, as simple as the chestnut tree 2 (Figure 3c) but with thinner branches. The pruned chestnut tree was selected for its more complex geometry than the previous ones: it is composed of more branches, which are less spaced, and which diameters are various (Figure 3d). The silver lime tree with shoots (Figure 3e) is a particular case, with a higher density of points. This case is interesting because it enables to assess the capacity of the algorithms for modeling intertwined and thin branches. Finally, the plane tree (Figure 3f) was picked to show if it is possible to reconstruct a tall tree with an important variation of branch diameters and an irregular positioning of branches. Overall, concerning the quality of the point clouds, the thinner the branches are and the taller the tree is, the greater the lack of information and noise on and around the branches are. A better setting of the TLS could improve the point clouds completeness, but with a greater acquisition time.

\begin{tabular}{|c|c|c|c|c|c|c|}
\hline & $\begin{array}{c}\text { Chestnut } \\
\text { tree } 1\end{array}$ & $\begin{array}{c}\text { Pruned } \\
\text { silver lime } \\
\text { tree }\end{array}$ & $\begin{array}{c}\text { Chestnut } \\
\text { tree } 2\end{array}$ & $\begin{array}{c}\text { Pruned } \\
\text { chestnut } \\
\text { tree }\end{array}$ & \begin{tabular}{|c|} 
Pruned \\
silver lime \\
tree with \\
shoots \\
\end{tabular} & $\begin{array}{c}\text { Pruned } \\
\text { plane tree }\end{array}$ \\
\hline $\begin{array}{c}\text { Tree } \\
\text { height }\end{array}$ & $6.0 \mathrm{~m}$ & $7.0 \mathrm{~m}$ & $7.9 \mathrm{~m}$ & $8.3 \mathrm{~m}$ & $9.2 \mathrm{~m}$ & $13.4 \mathrm{~m}$ \\
\hline $\begin{array}{l}\text { Trunk } \\
\text { height }\end{array}$ & $1.8 \mathrm{~m}$ & $2.2 \mathrm{~m}$ & $1.7 \mathrm{~m}$ & $3.7 \mathrm{~m}$ & $2.2 \mathrm{~m}$ & $3.1 \mathrm{~m}$ \\
\hline $\begin{array}{l}\text { Crown } \\
\text { shape }\end{array}$ & & $\Delta$ & & & & \\
\hline $\begin{array}{l}\text { Crown } \\
\text { volume }\end{array}$ & $3.0 \mathrm{~m}^{3}$ & $22.6 \mathrm{~m}^{3}$ & $47.4 \mathrm{~m}^{3}$ & $72.4 \mathrm{~m}^{3}$ & $84.1 \mathrm{~m}^{3}$ & $520.2 \mathrm{~m}^{3}$ \\
\hline $\begin{array}{l}\text { Crown } \\
\text { surface }\end{array}$ & $1.7 \mathrm{~m}^{2}$ & $11.3 \mathrm{~m}^{2}$ & $15.0 \mathrm{~m}^{2}$ & $24.5 \mathrm{~m}^{2}$ & $20.8 \mathrm{~m}^{2}$ & $75.7 \mathrm{~m}^{2}$ \\
\hline $\begin{array}{l}\text { Branch } \\
\text { density }\end{array}$ & $\begin{array}{l}\text { Low } \\
(23)\end{array}$ & $\begin{array}{l}\text { Low } \\
(56)\end{array}$ & $\begin{array}{l}\text { Middle } \\
\text { (296) }\end{array}$ & $\begin{array}{l}\text { Middle } \\
(131)\end{array}$ & $\begin{array}{l}\text { Large } \\
(\approx 975)\end{array}$ & $\begin{array}{c}\text { Large } \\
\text { (more than } \\
200)\end{array}$ \\
\hline $\begin{array}{c}\text { Branch } \\
\text { order }\end{array}$ & $\begin{array}{c}1-2 \\
\text { (shoots) }\end{array}$ & $1-2$ & 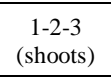 & $1-2-3$ & $\begin{array}{c}1-2-3 \\
\text { (shoots) }\end{array}$ & $\begin{array}{c}1-2-3-4 \ldots \\
\text { (shoots) }\end{array}$ \\
\hline $\begin{array}{c}\text { Branch } \\
\text { position }\end{array}$ & $\begin{array}{l}\text { Spaced } \\
\text { Not int. }\end{array}$ & $\begin{array}{c}\text { Spaced } \\
\text { Not int.* }\end{array}$ & $\begin{array}{c}\text { Close } \\
\text { Not int. }\end{array}$ & $\begin{array}{l}\text { Spaced } \\
\text { Not int. }\end{array}$ & $\begin{array}{l}\text { Close } \\
\text { Int. }\end{array}$ & $\begin{array}{c}\text { Close } \\
\text { Few int. }\end{array}$ \\
\hline \begin{tabular}{|c|} 
Branch \\
diameter
\end{tabular} & 1 to $5 \mathrm{~cm}$ & 5 to $15 \mathrm{~cm}$ & 1 to $8 \mathrm{~cm}$ & 2 to $15 \mathrm{~cm}$ & 1 to $15 \mathrm{~cm}$ & 5 to $30 \mathrm{~cm}$ \\
\hline
\end{tabular}

Table 2: Characteristics of selected trees $(*$ int. $=$ intertwined $)$. 


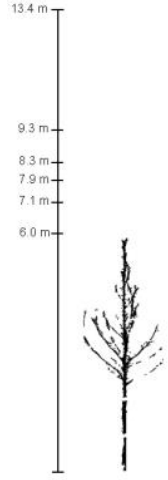

a)

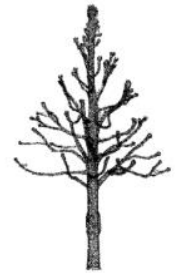

b)

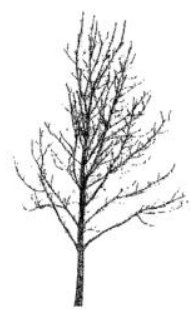

c)

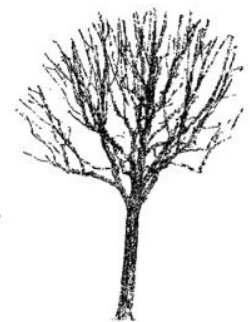

d)

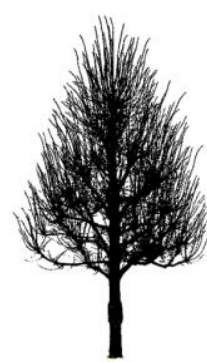

e)

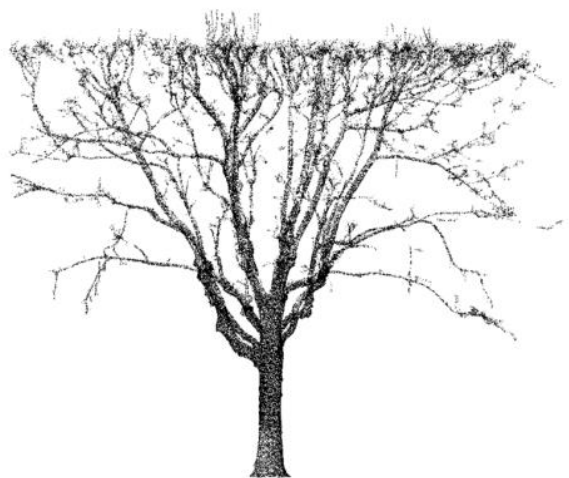

f)

Figure 3: Point clouds of several trees: a) a chestnut tree (1); b) a pruned silver lime tree; c) a chestnut tree (2); d) a pruned chestnut tree; e) a pruned silver lime tree with shoots and f) a pruned plane tree.

\section{RESULTS AND ANALYSIS}

\subsection{Results}

Table 5 illustrates the models produced by every algorithm for each tree under study. Based on these tree models, qualitative and quantitative assessments are performed.

\subsection{Qualitative assessment}

The first analysis consists in a visual inspection of the models produced by each algorithm and shown in Table 5. This manual process allows us to identify the strengths and drawbacks of each algorithm. Table 4 presents the conclusions of this analysis, according to several characteristics that we find relevant, such as the branch thickness, quantity and intersection or the amount of holes and noise in the point cloud. TreeArchitecture seems to be the most sensitive whereas PlanScan3D and SimpleTree are equivalent.

\begin{tabular}{|c|c|c|c|}
\hline Sensitivity & TreeArchitecture & PlantScan3D & SimpleTree \\
\hline Branch thickness & +++ & + & ++ \\
\hline Branch quantity & ++ & + & + \\
\hline Branch intersection & +++ & ++ & + \\
\hline Noise & +++ & ++ & + \\
\hline Holes & ++ & + & +++ \\
\hline
\end{tabular}

Table 4: Sensitivity of the algorithms (+ "few", ++ "normal" and +++ "strong") on several characteristics: branch thickness, quantity and intersections, noise and holes.

TreeArchitecture is the model which provides visually the poorest results when the tree geometry is not simple, e.g. case of the pruned silver lime tree with shoots in Table 5. Thin branches (about $2 \mathrm{~cm}$ ) are rarely reconstructed. Errors of reconstruction occur in the case of noisy data and lots of branches or when there are intersections between branches. Some branches are not connected to the other ones. Moreover, most of the time, the volume is overestimated and this phenomenon is worse with thin branches. However, this algorithm contains some advantages: generally, the exact extremity of branches are reconstructed. Moreover cylinders used for the volume estimation are linked to the others.

PlantScan3D is the algorithm which detects the most branches even with a large range of diameters (from $2 \mathrm{~cm}$ to $30 \mathrm{~cm}$ ). The model quality is not affected by an important quantity of branches. Intersections between branches create some errors in reconstruction, but less than with the previous algorithm. All branches are connected to each other, but they are slightly shortened at their extremities. Concerning the quality of the point cloud, holes have no influence on the reconstruction, in contrary to noise. Indeed, several parts of branches are detected twice. This happens most of the time in presence of noise, or thin branches, or connections with short branches. Moreover, the wood volume estimated by the PlantScan3D seems better than the volume estimated with TreeArchitecture, despite some overestimations at branch junctions. Finally, this is the only algorithm using cones, this is the reason why it offers a smooth and realistic model, and also improves the volume determination.

With SimpleTree, there are no errors of reconstruction, only missing branches. As with the previous algorithm, branches are always connected to the others, but there is also an underestimation of the branch length at their extremities. Most of the diameters of the branches are detected, but thin branches with few points are less well detected than with PlantScan3D. This algorithm is the best for taking into account branch intersections, even if some cases of intersections are not well reconstructed. While noise has little impact on the model, this algorithm is very sensitive to holes. As we can see in the case of the pruned chestnut tree in Table 5, the branches are shorter than observed in the point cloud because of holes. Concerning the volume of the wood, it is slightly overestimated or underestimated, but it seems better than the volume estimated with the other reconstruction algorithms. However, due to a few holes between the cylinders, which are not always linked, the total branch length and volume is slightly underestimated.

Regarding the comprehensibility of the output, PlantScan3D offers a well-organized branch structures graph, in which shoots are considered as branches. TreeArchitecture provides a file where each node is classified by branch, but the branch order information is lost. Finally, with SimpleTree, the topology information is not lost and a classification of each point following branches or segments composing the branches is achieved. However, with this classification, branches are not classified logically, e.g. one branch skeleton represents a group of several branches, or one branch skeleton is only a part of the real branch. 


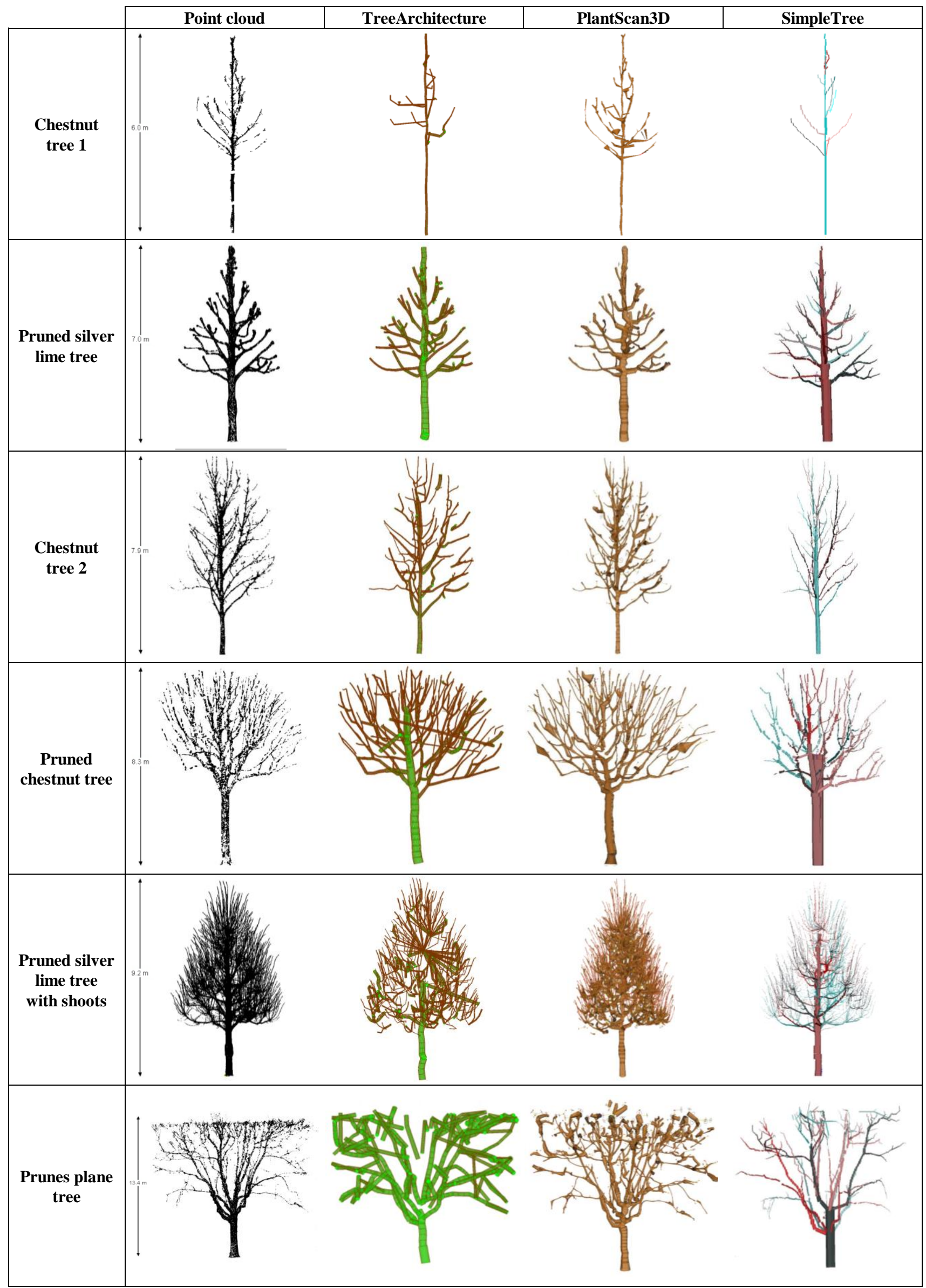

Table 5: 3D models of tree point clouds, produced with TreeArchitecture, PlantScan3D and SimpleTree. 


\begin{tabular}{|c|c|c|c|c|c|c|c|c|c|c|}
\hline & \multicolumn{6}{|c|}{ Number of detected branches } & \multicolumn{4}{|c|}{ Total length of branches (m) } \\
\hline & Ref & M1a & M1b & M2a & M2b & M3 & Ref & M1 & M2 & M3 \\
\hline Chestnut tree 1 & 23 & $\begin{array}{c}13 \\
(57 \%)\end{array}$ & $\begin{array}{c}12 \\
(52 \%)\end{array}$ & $\begin{array}{c}88 \\
(383 \%)\end{array}$ & $\begin{array}{c}20 \\
(87 \%)\end{array}$ & $\begin{array}{c}23 \\
(100 \%)\end{array}$ & 28.1 & $\begin{array}{c}11.8 \\
(42 \%)\end{array}$ & $\begin{array}{c}33.7 \\
(121 \%)\end{array}$ & $\begin{array}{c}15.1 \\
(54 \%)\end{array}$ \\
\hline Pruned silver lime tree & 56 & $\begin{array}{c}52 \\
(93 \%)\end{array}$ & $\begin{array}{c}53 \\
(95 \%)\end{array}$ & $\begin{array}{c}60 \\
(107 \%)\end{array}$ & $\begin{array}{c}52 \\
(93 \%)\end{array}$ & $\begin{array}{c}52 \\
(93 \%)\end{array}$ & 68.1 & $\begin{array}{c}52.8 \\
(78 \%)\end{array}$ & $\begin{array}{c}61.0 \\
(90 \%)\end{array}$ & $\begin{array}{c}62.9 \\
(92 \%)\end{array}$ \\
\hline Chestnut tree 2 & 296 & $\begin{array}{c}67 \\
(23 \%)\end{array}$ & $\begin{array}{c}70 \\
(24 \%)\end{array}$ & $\begin{array}{c}198 \\
(67 \%)\end{array}$ & $\begin{array}{c}145 \\
(49 \%)\end{array}$ & $\begin{array}{c}81 \\
(27 \%)\end{array}$ & 174.0 & $\begin{array}{c}89.2 \\
(51 \%)\end{array}$ & $\begin{array}{c}135.3 \\
(78 \%)\end{array}$ & $\begin{array}{c}90.8 \\
(52 \%)\end{array}$ \\
\hline Pruned chestnut tree & 131 & $\begin{array}{c}110 \\
(84 \%)\end{array}$ & $\begin{array}{c}108 \\
(82 \%)\end{array}$ & $\begin{array}{c}202 \\
(154 \%)\end{array}$ & $\begin{array}{c}124 \\
(95 \%)\end{array}$ & $\begin{array}{c}70 \\
(53 \%)\end{array}$ & 231.2 & $\begin{array}{c}178.2 \\
(77 \%)\end{array}$ & $\begin{array}{c}223.5 \\
(97 \%)\end{array}$ & $\begin{array}{c}112.6 \\
(49 \%)\end{array}$ \\
\hline $\begin{array}{r}\text { Pruned silver lime tree with } \\
\text { shoots }\end{array}$ & 976 & $\begin{array}{c}325 \\
(33 \%)\end{array}$ & I & $\begin{array}{c}1326 \\
(136 \%)\end{array}$ & l & $\begin{array}{l}\approx 1089 \\
(112 \%)\end{array}$ & 1026.9 & $\begin{array}{l}401.7 \\
(39 \%)\end{array}$ & $\begin{array}{l}925.5 \\
(90 \%)\end{array}$ & $\begin{array}{l}697.9 \\
(68 \%)\end{array}$ \\
\hline Pruned plane tree & I & 61 & / & 511 & / & $\approx 194$ & / & 319.6 & 478.5 & 239.8 \\
\hline
\end{tabular}

Table 6: Assessment of the number of branches and of the total length of branches reconstructed with TreeArchitecture (M1), PlantScan3D (M2) and SimpleTree (M3).

\begin{tabular}{|c|c|c|c|c|c|c|c|}
\hline & \multicolumn{4}{|c|}{ DBH (cm) } & \multicolumn{3}{|c|}{ Volume $\left(\mathbf{m}^{3}\right)$} \\
\hline & Ref & M1 & M2 & M3 & M1 & M2 & M3 \\
\hline Chestnut tree 1 & 8 & $\begin{array}{c}6 \\
(75 \%)\end{array}$ & $\begin{array}{c}7 \\
(88 \%)\end{array}$ & $\begin{array}{c}5 \\
(63 \%)\end{array}$ & 0.03 & 0.04 & 0.02 \\
\hline Pruned silver lime tree & 38 & $\begin{array}{c}28 \\
(74 \%)\end{array}$ & $\begin{array}{c}36 \\
(95 \%)\end{array}$ & $\begin{array}{c}32 \\
84 \%)\end{array}$ & 0.70 & 0.71 & 0.62 \\
\hline Chestnut tree 2 & 16 & $\begin{array}{c}16 \\
(100 \%)\end{array}$ & $\begin{array}{c}15 \\
(94 \%)\end{array}$ & $\begin{array}{c}16 \\
(100 \%)\end{array}$ & 0.32 & 0.61 & 0.25 \\
\hline Pruned chestnut tree & 35 & $\begin{array}{c}32 \\
(91 \%)\end{array}$ & $\begin{array}{c}32 \\
(91 \%)\end{array}$ & $\begin{array}{c}34 \\
(97 \%)\end{array}$ & 0.99 & 0.92 & 1.02 \\
\hline $\begin{array}{r}\text { Pruned silver lime tree with } \\
\text { shoots }\end{array}$ & 38 & $\begin{array}{c}28 \\
(74 \%)\end{array}$ & $\begin{array}{c}36 \\
(95 \%)\end{array}$ & $\begin{array}{c}32 \\
(84 \%)\end{array}$ & 1.14 & 3.92 & 0.57 \\
\hline Pruned plane tree & 61 & $\begin{array}{c}56 \\
(92 \%)\end{array}$ & $\begin{array}{c}60 \\
(98 \%)\end{array}$ & $\begin{array}{c}64 \\
(105 \%)\end{array}$ & 23.02 & 11.29 & 4.19 \\
\hline
\end{tabular}

Table 7: Assessment of the extracted Diameters at Breast Height (DBH) and of the determined volumes of the wood obtained with TreeArchitecture (M1), PlantScan3D (M2) and SimpleTree (M3).

\subsection{Quantitative assessment}

Given the presented qualitative analysis, we can state that the three reconstruction algorithms make the realistic modeling of different complex geometries of trees possible, even if some errors appear in the reconstructions.

In this subsection, the quantitative assessment of the results is carried out. This step consists in comparing, for each algorithm, several extracted tree characteristics which are considered as relevant in a tree model. As detailed in Table 6 and in Table 7 the tree characteristics are the number of branches, the length of branches, the Diameter at Breast Height (DBH) and the volume of the wood. These characteristics are given by the algorithms, except for the number of branches with SimpleTree. The automatic classification of branches is not exact, as described in subsection 4.2. Therefore, we manually accounted for the detected branches (results M3 in Table 6) by superimposing SimpleTree models with the corresponding point cloud. Moreover, with the same operations we recorded, for every model and every algorithm, the number of well reconstructed branches (results M1b and M2b in Table 6) which is different from the number of branches created by the algorithms (results M1a and M2a in Table 6).
Then, the extracted values, such as the length, the number of branches and the DBH are compared with the reference values. The volume of the wood is not considered, because we have not the means to perform these ground-truth measurements. For the whole of the trees, excepted for the plane tree, reference skeletons were created. This enables the validation of the reconstructed trees on characteristics, as the number and length of branches. For the pruned silver lime tree with shoots, the reference model was created by manual digitalization of the shoots on the point cloud. Reference skeletons of the other trees were obtained by using the PlantScan3D module, allowing manual modifications of a first raw skeleton automatically created. These processes were fastidious but they allow to produce reference models which are required to assess the accuracy of the created models. The DBH estimation is compared to manual field measurements on trees at 1.3 meter height.

From the skeleton analysis of each model (Table 6), we can affirm that the algorithm which reconstructs most of the branches is PlantScan3D (M2b). However, a solution to remove the duplicate errors (explained in subsection 4.2) that this algorithm creates (M2a) must be found. TreeArchitecture (M1) and SimpleTree (M3) are equivalent regarding the detection of branches. This observation does not mean that the reconstructions are always correct, as we saw in the qualitative assessment part. Regarding the results obtained for length 
estimations, again PlantScan3D seems to provide the best results, because it reconstructs most of the branch lengths. However, as PlantScan3D models leads to small duplicate errors, the total length of branches is slightly overestimated. Regarding the volumetric part of the models, as we can see in Table 7 , the DBH is accurately determined by all algorithms, but slightly better with SimpleTree. Unfortunately we do not have reference values for the volume of the biomass. However, we can observe that TreeArchitecture and PlantScan3D usually overestimate the biomass volume compared to SimpleTree. The SimpleTree algorithm of volume estimations was assessed by the developers with ground-truth measurements for 36 trees and it is based on an assessed allometric rule saying that the cross sectional area before a branch junction equals the sum of the cross sectional areas after the branch junction (Hackenberg et al., 2015). Therefore, we can consider that SimpleTree estimations of the tree wood are reliable.

\section{DISCUSSION}

Based on the analysis presented in the previous section, it is possible to carry out a global conclusion on the possibility of modeling trees with different geometries. For a simple geometry, such as with the pruned silver lime tree, all algorithms provide realistic results. However, when the geometry becomes more complex the reconstruction becomes less accurate. The reconstruction of one tree with different diameters of branches is possible, but the thinner are the branches, the worse is the model, in terms of branch detection and also branch volume.

This is due to the quantity of points on the branch against its diameter, i.e. around 40 points for a diameter of $2 \mathrm{~cm}, 90$ points for $5 \mathrm{~cm}$ and 190 points for $15 \mathrm{~cm}$. But, this density of points depends on the TLS setting. With a TLS setting of a $6 \mathrm{~mm}$ spacing between points at $10 \mathrm{~m}$, the reconstructions succeed (case of the pruned silver lime tree with shoots of $1 \mathrm{~cm}$ in diameter) whereas with $12 \mathrm{~mm}$ it is not always the case (case of the chestnut tree 1 and 2). However, this difference of point density has no impact on the skeleton reconstruction of branches with minimum diameters of $5 \mathrm{~cm}$. The same phenomenon is observed with branch intersections. Moreover, following the geometric complexity of trees and the number of TLS stations recorded around trees, the point distribution in the point cloud can be not uniform. Depending on the reconstruction algorithms, the point distribution will have more or less impacts on the skeleton and on a good volume estimation. Finally, when there are lots of intersections and a large amount of branches (case of the pruned silver lime tree with shoots) none of the methods delivers accurate results. This is the common limit of the tested reconstruction algorithms.

Other important criteria to analyze are the use and the performance of the algorithms. Firstly, TreeArchitecture does not provide an ergonomic user interface unlike the others and PlantScan3D needs only two parameters, whereas the others require 17 parameters. For these reasons, PlantScan3D is user friendlier. Secondly, the processing time is the shortest with PlantScan3D. For a processor Intel Core I7, with a 16 Go RAM, 5 minutes are necessary to reconstruct a tree of 700,000 points and few seconds for 50,000 points. For the same number of points, TreeArchitecture needs 10 hours and 15 minutes and SimpleTree needs 4 hours and 5 minutes.
It is important to notice that the developers of PlantScan3D and SimpleTree are continuously improving their algorithm. During our study, new versions emerged. A better skeletonization method (Preuksakarn, 2012) will be soon included in the open source tool, PlantScan3D. Concerning SimpleTree, a new version appeared, requiring no parameters and available on the Windows operating system. This new version of SimpleTree should certainly resolve reconstruction errors due to a wrong choice of parameters by the user.

\section{CONCLUSION}

In this paper, we assessed the possibility of accurately reconstructing various geometries of tree woods, representing the diversity of trees in the urban environment. This evaluation was combined with the comparison of three different algorithms of reconstruction. This study helped us to choose the best algorithm for tree wood reconstruction.

Six trees have been chosen, with different geometries, to carry out this assessment study. Tree sizes are between 6 meters and 13.4 meters high, with branch diameter between 2 centimeters and 30 centimeters. In this sample, there are spaced branches and close branches but also intertwined branches. Each tree has been reconstructed with three different algorithms dedicated to 3D reconstruction of trees: TreeArchitecture, PlantScan3D and SimpleTree. These algorithms use different methods to operate and have different goals. For all of them, the produced 3D tree models are composed of a skeleton and cylinders or cones. A qualitative assessment by comparing tree point clouds and a quantitative assessment that compared automatic and manual tree reconstructions have been performed. The strengths and the drawbacks of the algorithms were highlighted. PlantScan3D is the model which detects most of the branches and estimates correctly their length, whereas SimpleTree seems better for the tree wood volume determination. But, with both algorithms, different errors of reconstruction appear, in particular when branches are numerous, intertwined and thin. This type of tree geometry is considered as the limit of having an accurate reconstruction with tree reconstruction algorithms. Concerning TreeArchitecture, results are accurate with simple tree geometries only.

In the context of our climatological study, our aim is to estimate the cooling effect of trees on the urban climate by the evapotranspiration rate which is closely related to the leaf surface distribution in the tree crown. The accuracy of the reconstructed foliage depends on the accuracy of the skeleton of the tree used as input. Based on the analysis made in this study, we are able to extract automatically an accurate tree skeleton from a TLS point cloud. Now, in order to determine if the tree skeleton obtained from the previous algorithms are sufficient for the estimation of the tree evapotranspiration rate with the ecophysiological model, leaves will be reconstructed and attached to vegetative shoots by using allometric statistics, as it was made in (Bournez et al., 2016). In the future, we would like to reconstruct several trees simultaneously, using for instance data provided by terrestrial mobile laser scanners, in order to simulate the evapotranspiration rate for several trees simultaneously. 


\section{ACKNOWLEDGEMENTS}

The authors would like to thank Marine Lempereur, INSA master student, for performing the reconstruction of the trees with the three algorithms and also Frédéric Boudon, INRIA member, and Jan Hackenberg, INRA member, for providing help, respectively for the use of PlantScan3D and SimpleTree.

The authors would also like to thank the Eurometropolis of Strasbourg for providing them access to 2D and 3D data over Strasbourg.

\section{REFERENCES}

Boudon, F., Preuksakarn, C., Ferraro, P., Diener, J., Nacry, P., Nikinmaa, E., and Godin, C., 2014. Quantitative assessment of automatic reconstructions of branching systems obtained from laser scanning. Annals of Botany, 114(4), pp. 853-862.

Bournez, E., Landes, T., Saudreau, M., Kastendeuch, P., and Najjar, G., 2016. Impact of level of details in the 3D reconstruction of trees for microclimate modeling. In: ISPRS International Archives of the Photogrammetry, Remote Sensing and Spatial Information Sciences, Prague, Czech Republic, pp. 257-264.

Bucksch, A., Lindenbergh, R., and Menenti, M., 2010. Robust skeleton extraction from imperfect point clouds. Visual Computer, 26(10), pp. 1283-1300.

Cao, J., Tagliasacchi, A., Olson, M., Zhang, H., and Su, Z., 2010. Point cloud skeletons via Laplacian-based contraction. In: Proceedings of the IEEE International Conference on Shape Modeling and Applications, Aix en Provence, France, pp.187197.

Cornea, N.D., Silver, D., Yuan, X., and Balasubramanian, R., 2005. Computing hierarchical curve-skeletons of 3D objects. Visual Computer, 21(11), pp. 945-955.

Côté, J.F., Widlowski, J.L., Fournier, R.A., and Verstraete, M.M., 2009. The structural and radiative consistency of threedimensional tree reconstructions from terrestrial lidar. Remote Sensing of Environment, 113(5), pp. 1067-1081.

Grussenmeyer, P., Landes, T., Doneus, M., and Lerma, J.L., 2016. Basics of range-based modelling techniques in Cultural Heritage. In: Remondino, F., Stylianidis, S. (Eds.), 3D Recording, Documentation and Management in Cultural Heritage. Whittles Publishing, Dunbeath, Caithness, Scotland, pp. 305-368.

Hackenberg, J., Morhart, C., Sheppard, J., Spiecker, H., and Disney, M., 2014. Highly accurate tree models derived from terrestrial laser scan data: A method description, Forests, 5(5), pp. 1069-1105.

Hackenberg, J., Spiecker, H., Calders, K., Disney, M., and Raumonen, P., 2015. SimpleTree - An efficient open source tool to build tree models from TLS clouds, Forests, 6(11), pp. 42454294.

Landes, T., Saudreau, M., Najjar, G., Kastendeuch, P., Guillemin, S., Colin, J., and Luhahe, R., 2015. 3D tree architecture modeling from laser scanning for urban microclimate study. In: Proceedings of the 9th International Conference on Urban Climate jointly with 12th Symposium on the Urban Environment, Toulouse, France.
Livny, Y., Yan, F., Olson, M., Chen, B., Zhang, H., and El-Sana, J., 2010. Automatic Reconstruction of Tree Skeletal Structures from Point Clouds. ACM Transactions on Graphics, Proceedings of ACM SIGGRAPH, Asia. 29(6).

Pfeifer, N., Gorte, B., Winterhalder, D., Sensing, R., and Range, C., 2004. Automatic Reconstruction of Single Trees from Terrestrial Laser Scanner Data. In: ISPRS International Archives of the Photogrammetry, Remote Sensing and Spatial Information Sciences, Istanbul, Turkey, pp. 114-119.

Preuksakarn, C., 2012. Reconstructing Plant Architecture from 3D Laser Scanner Data. Thesis, Montpellier 2 University, in France.

Quan, L., Tan, P., Zeng, G., Yuan, L., Wang, J., and Kang, S.B., 2006. Image-based Plant Modeling. ACM Transactions on Graphics, Proceedings of ACM SIGGRAPH, Asia, 25(3), pp. 599-604.

Rahman, M.Z.A., Majid, Z., Bakar, M.A.A., Rasib, A.W., and Kadir, W.H.W., 2015. Individual tree measurement in tropical environment using terrestrial laser scanning. Jurnal Teknologi, 73(5), pp. 127-133.

Raumonen, P., Kaasalainen, M., Åkerblom, M., Kaasalainen, S., Kaartinen, H., Vastaranta, M., Holopainen, M., Disney, and M., Lewis, P., 2013. Fast Automatic Precision Tree Models from Terrestrial Laser Scanner Data. Remote Sensing, 5(2), pp. 491520

Sinoquet, H., Le Roux, X., Adam, B., Ameglio, T., and Daudet, F.A., 2001. RATP: a model for simulating the spatial distribution of radiation absorption, transpiration and photosynthesis within canopies: application to an isolated tree crown. Plant, Cell \& Environment, 24, pp. 395-406.

Sinoquet, H., and Rivet, P., 1997. Measurement and visualization of the architecture of an adult tree based on a threedimensional digitising device. Trees, 11(5), pp. 265-270.

van Leeuwen, M., and Nieuwenhuis, M., 2010. Retrieval of forest structural parameters using LiDAR remote sensing. European Journal of Forest Research, 129(4), pp. 749-770.

$\mathrm{Xu}$, H., Gossett, N., and Chen, B., 2007. Knowledge and Heuristic Based Modeling of Laser-Scanned Trees. ACM Transactions on Graphics, 26(4). 